\title{
Evaluation of FEC for the Atmospheric Optical IM/DD Channel
}

\author{
Hennes Henniger, Florian David, Dirk Giggenbach ${ }^{\text {a }}$ \\ Christoph Rapp ${ }^{\mathrm{b}}$ \\ ${ }^{a}$ German Aerospace Center (DLR), Oberpfaffenhofen; \\ ${ }^{\mathrm{b}}$ Munich University of Applied Sciences
}

\begin{abstract}
Channel measurements were performed by the German Aerospace Center in various near ground optical channels including a $1.5 \mathrm{~km}$ horizontal path as well as a $61 \mathrm{~km}$ path. These measurements clearly showed that the atmosphere causes very slow fading (compared to the high data rates usually used in optical communication systems), which significantly degrades the transmission quality. As transmitter power and receiver sensitivity are limited by the transmission technology, fading depicts a severe problem, that can be reduced by the use of forward error correction schemes (FEC) in order to improve system performance. Therefore FEC was subject of investigations by means of simulations. To figure out which FEC methods are useful for applications in the atmospheric optical channels simulations of standard block codes and interleavers have been done. They were based on data sets taken in the various channel measurements. The simulations point out that only very long interleaving can increase performance significantly.
\end{abstract}

Keywords: atmospheric optical transmission, optical fading channel, IM/DD, coding, interleaving

\section{INTRODUCTION}

In the near ground atmospheric optical channel the received power fluctuates due to index of refraction turbulence. This effect of intensity variations is called scintillation, this means that the incoming light is affected by fades and also by surges. In general we have to deal with deep fades and also with high atmospheric attenuation in the boundary layer. The fade time mainly depends on the weather situation (especially wind speed and sunshine) and usually the fading time constant is much bigger than one bit duration.

One way to overcome the fading is a very large link margin which is only applicable for very short distances, because the laser transmitter power is limited by technology and also by eye safety regulations. Another way is to benefit from channel coding. In this work we only care about straight forward error correction schemes and interleaving techniques. These methods are better suited to the high data rate communication systems in fading channels, because FEC coding schemes provide a higher information flow rate than e.g. automatic repeat request (ARQ) schemes. Usually, for investigations of FEC a channel model is used, but for the atmospheric optical fading channel, there is not yet a model available, which fits all scenarios very well. Hence we tried to characterise two typical channels using measurement data and then based our simulations on these channel measurements.

\footnotetext{
Author Contact: hennes.henniger@dlr.de

Hennes Henniger, Institute of Communications and Navigation,

German Aerospace Center (DLR), Site Oberpfaffenhofen, D-82234 Wessling, Germany

Copyright 2003 Society of Photo-Optical Instrumentation Engineers.

This paper was published in Proc. of SPIE Vol. 4975 and is made available as an electronic reprint with permission of SPIE. One print or electronic copy may be made for personal use only. Systematic or multiple reproduction, distribution to multiple locations via electronic or other means, duplication of any material in this paper for a fee or for commercial purposes, or modification of the content of the paper are prohibited.
} 


\section{CHANNEL STATISTICS OF A TYPICAL ATMOSPHERIC OPTICAL FADING CHANNEL}

\subsection{Statistical parameters to compare the channel behaviour}

The German Aerospace Center (DLR) performed channel measurements in various atmospheric optical channels, e.g. during the Wallberg experiment (WB) in the frame of the FASOLT project $[2,3,5]$. A CW laser diode transmitter at $980 \mathrm{~nm}$ was placed on a mountain top in the German Alps at a height of about $1620 \mathrm{~m}$. The receiver was located at the DLR site Oberpfaffenhofen near Munich at a height of $620 \mathrm{~m}$. The receiver consists of an achromat $(\mathrm{D}=75 \mathrm{~mm}$, $\mathrm{f}=300 \mathrm{~mm}$ ) focusing the incoming light to a $200 \mu \mathrm{m}$ multimode fiber. Background light was blocked by an optical filter. The received power was measured with a fiber coupled optical power meter and recorded using an ADC and sampling equipment. The optical path length between Wallberg and Oberpfaffenhofen is about $61 \mathrm{~km}$. Additional information about this scenario and the FASOLT experiment can be found in [5],[2] and [3].

Another test path was installed at the airport Oberpfaffenhofen (OP). Here, the transmitter consisted of a $808 \mathrm{~nm} \mathrm{CW}$ laser diode. For the receiver the same equipment as in the Wallberg (WB) experiment was used. The laser beam was propagating very close to ground, passing grass and asphalt areas with a total path length of $1.5 \mathrm{~km}$. In both scenarios (WB, OP), neither for transmitter nor for the receiver active pointing or tracking was used.

For each channel measurement the received power is sampled by ADCs and stored into a power vector. From these vectors statistical parameters are calculated to compare the measurements and scenarios and to characterize the channel behaviour. It can be e.g. described with by probability of fades (POF) for an $-\mathrm{x} \mathrm{dB}$ fade according to the following equation:

$$
\begin{aligned}
\operatorname{POFx} d B=\frac{1}{n} \sum_{i=1}^{n}\left[P_{i}<\langle P\rangle \cdot 10^{-0.1 \cdot x}\right] \\
\text { with: } \quad \mathrm{n} \quad \text { length of the power vector, total number of samples } \\
<\mathrm{P}>\quad \text { mean received power } \\
\mathrm{P}_{\mathrm{i}} \quad \text { element } \mathrm{i} \text { of the power vector }
\end{aligned}
$$

The POF reflects the occurance of signal levels below a given threshold -x dB.

Furthermore the mean duration of fades $(<\mathrm{DOFxdB}>)$ can be computed from the power vector. It describes the time behaviour of the fading by the mean time a fade stays below a certain $-\mathrm{x} \mathrm{dB}$ level.

From the auto correlation function the full width half maximum time $(\tau)$ is calculated to approximately specify the power density spectrum of the received power. $\tau$ was calculated to $280 \mathrm{msec}$ for the Wallberg scenario and $4.0 \mathrm{msec}$ for Oberpfaffenhofen respectively.

Also the power scintillation index (PSI) is used to compare the channels to each other. It is the normalized variance of the received power. It is defined as:

$$
P S I=\frac{\left\langle P_{i}^{2}\right\rangle}{\left\langle P_{i}\right\rangle^{2}}-1
$$

The power scintillation index was calculated for the Wallberg experiment to about 0.29 and for the Oberpfaffenhofen path to 0.25 . It depends on the aperture diameter $\mathrm{D}$, which was the same for all measurements.

\subsection{Comparing channel measurements}

In the following there are some plots, showing the statistical parameters described above. They include histograms of received optical power as well as mean duration and probability of fade. These plots are selected examples of data taken during the measurements in the Wallberg (WB) and Oberpfaffenhofen (OP) scenario. The coding simulations described in the following chapters are all based on these two selected channel measurements. 

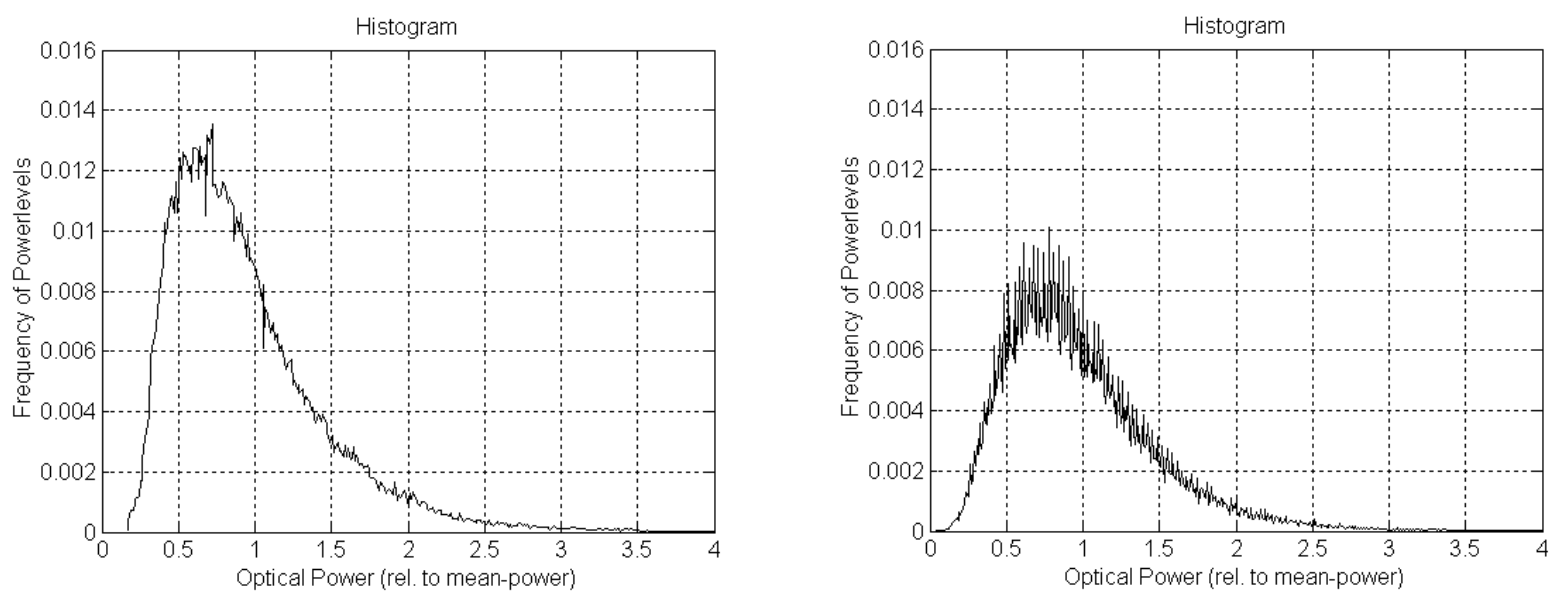

Figure 1: Normalized histogram of the received power for WB (left) and OP (right)
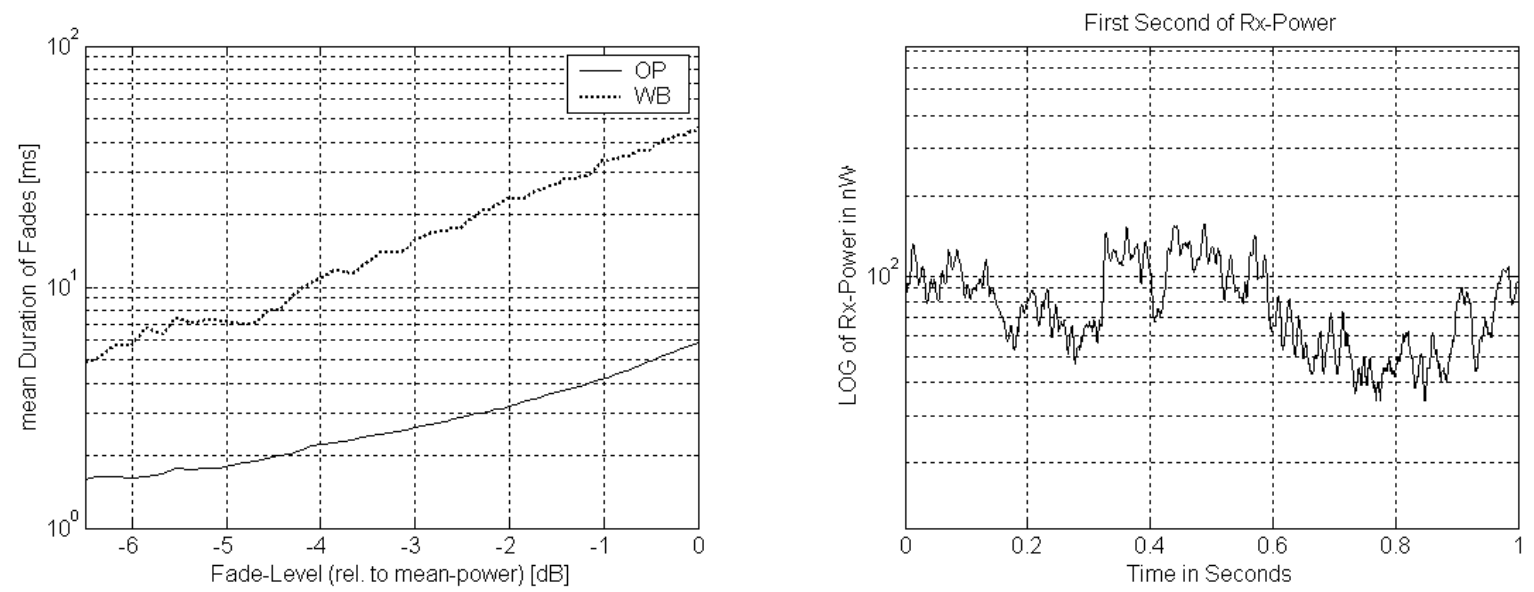

Figure 2: mean duration of fade vs. fade level $[\mathrm{dB}]$ for WB and OP (left), time-plot of the received power for the first second of the selected measurement in the WB scenario (right)
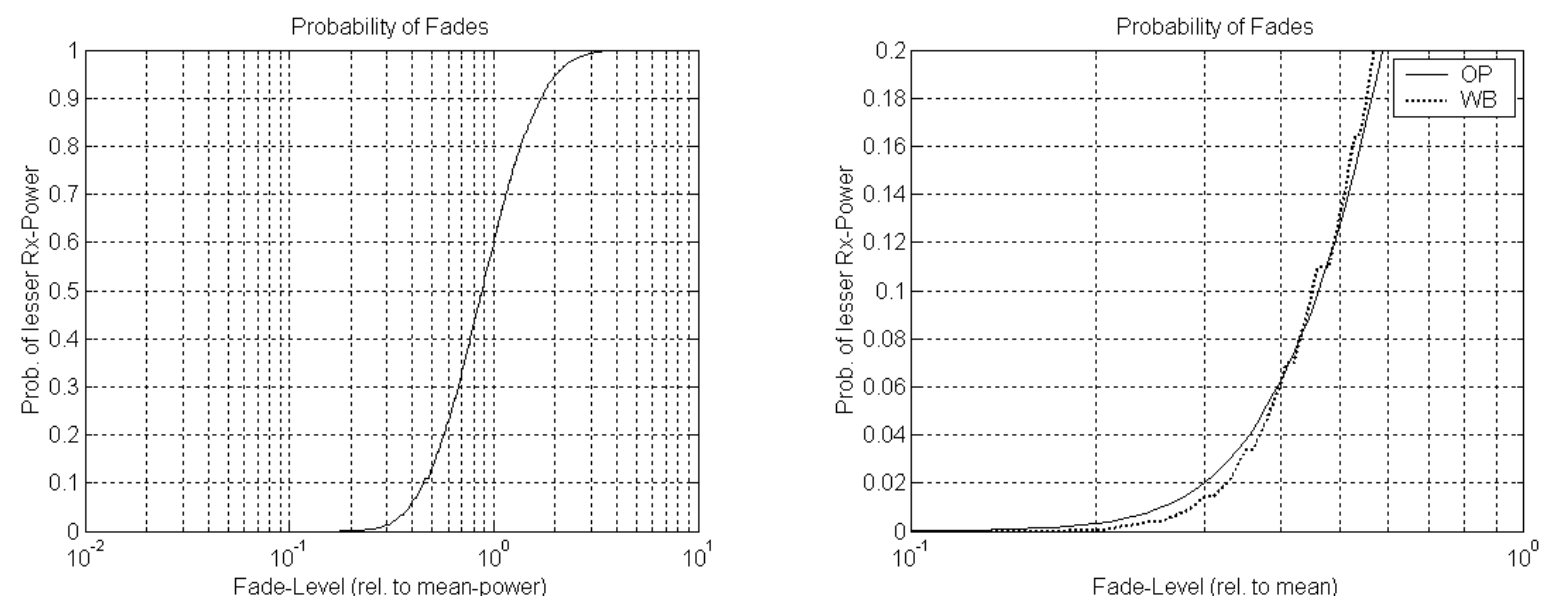

Figure 3: probability of fades vs. fade level in the WB scenario (left), probability of fade vs. fade level for WB and OP (right) 
As figure 1 shows, the histograms for the Wallberg (WB) and Oberpfaffenhofen (OP) scenario are quite similar, but the fading frequency as characterised by the mean duration of fade (displayed in figure 2) is very different. The longer fade duration correlates with the longer full width half maximum time $\tau$ of the auto-correlation of the received power. It is obvious that the time constants of the channel are orders of magnitude bigger than one bit duration when using high data rates $(\geq 100 \mathrm{Mbps})$. Hence the channel can be described as a very slow fading channel. Characterising the optical fading channel with synonyms from the microwave channel behaviour description, the optical channel is also non frequency selective. Furthermore the channel has a very long memory, because the channel state is constant for a very long time. The error probability of each symbol depends on past symbols, since the expected received power can be assumed as constant for a very large amount of symbols. When receiving constant power, the signal is only affected by the detector noise.

\section{THEORY OF BLOCK CODING AND INTERLEAVING}

\subsection{Block Codes}

Block codes add a certain amount of redundancy to a block of information bits. The encoder of a socalled (n,k) block code transforms a message of $\mathrm{k}$ symbols into a codeword of $\mathrm{n}>\mathrm{k}$ symbols. In a block code, each codeword depends only on the current input information, but not on preceding codewords. Therefore the code is called memoryless. In this work we discuss only codes with binary symbols, since they are most often used due to the lower implementation complexity. The code rate $\mathrm{R}$ determines the amount of redundancy and is defined as:

$R=\frac{k}{n}$

If we consider an linear block code, the maximum number of correctable errors $t$ is given by:

$t \leq\left\lfloor\frac{n-k}{2}\right\rfloor$

Equation (4) gives a theoretically limit only. However, it is not sure that such a code really exists. Typical codes used in communication systems can be found e.g. in [4].

In a communication system the receiver decides whether the received signal corrupted by noise resembles a zero or a one bit. In block codes usually hard decision is used, because soft decision is too computationally expensive for block codes, since commonly used block length higher than 128bit. For hard decision, there are different concepts to decide which codeword was sent. A very common concept is the bounded minimum distance (BMD) concept.

In the BMD concept, there is a certain probability of identifiable, but not corrigible errors. If the number of errors in the codeword is much higher than the number of correctable bits $t$, the received word may resemble to a codeword other than the sent one. Such errors are undetectable. The mean probability $p_{u e}$ for receiving a word with undetectable errors over the ensemble of all linear $(\mathrm{n}, \mathrm{k})$ block codes, depending on the error rate of the channel $\mathrm{p}_{\mathrm{bit}, \mathrm{cod}}$, is given by [1]:

$\left\langle p_{\text {ue }}\right\rangle=\left[1-\left(1-p_{\text {bit }, \text { cod }}\right)^{k}\right] 2^{-(n-k)}$

It can be shown that the block error probability $\mathrm{p}_{\mathrm{bl}}$ for long codes $(\mathrm{n}>128)$ is dominated by the probability of receiving non correctable words. Hence, for BMD decoding the block error probability $\mathrm{p}_{\mathrm{bl}}$ of $\mathrm{a}(\mathrm{n}, \mathrm{k})$ block code can be calculated ,depending on the bit error probability of the channel $\mathrm{p}_{\text {bit,cod }}$ (for a transmission rate which is a factor of $1 / \mathrm{R}$ higher than the message data rate) from [4]:

$p_{b l}=\sum_{e=t+1}^{n}\left(\begin{array}{l}n \\ e\end{array}\right) p_{b i t, c o d}^{e}\left(1-p_{b i t, c o d}\right)^{n-e}$ 
For the bit error probability $\mathrm{p}_{\mathrm{bit}, \mathrm{dec}}$ only an upper bound can be given:

$$
p_{\text {bit.dec }} \leq \sum_{e=t+1}^{n} \min \left\{1, \frac{e+t}{k}\right\}\left(\begin{array}{l}
n \\
e
\end{array}\right) p_{b i t, c o d}^{e}\left(1-p_{b i t, c o d}\right)^{n-e}
$$

This upper bound is used in a quasi-analytically simulation model, which will be described in chapter 4 . Based on the measured data sets, for each instantaneous value of channel error probability (depending on the received power) a value for the bit error probability after decoding will be calculated using formula (7).

\subsection{Interleavers}

To spread long burst errors and to avoid having more than the maximum number of correctable errors in one codeword, interleaving is used. Interleaving is a process of rearranging the order of a data sequence in a deterministic format. The inverse process is called deinterleaving and is used to restore the original order of the sequence in the receiver. A simple concept is the technique of block interleaving, where serial data is written row-wise into the interleaver matrix and read out column-wise as illustrated in figure 4 . The number of columns is denoted as $\mathrm{N}$, the number of rows as $\mathrm{M}$ respectively. $\mathrm{M}$ is also referred to as interleaver depth. For a block interleaver, a storage device of $\operatorname{size} N \cdot M$ is needed. As a result of interleaving, there is a constant end to end delay in the communication system of

$\Delta t=2 N M T$

where $\mathrm{T}$ is the bit duration. As figure 4 shows, the block interleaver concept fails to break low weight error patterns. Block deinterleavers are only effective, if the error patterns to be broken are confined to one row [9].

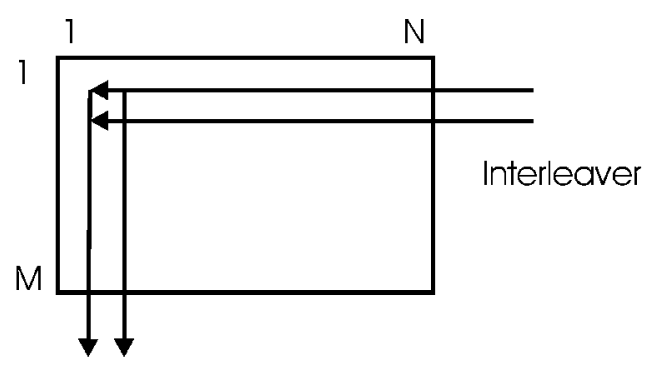

Channel

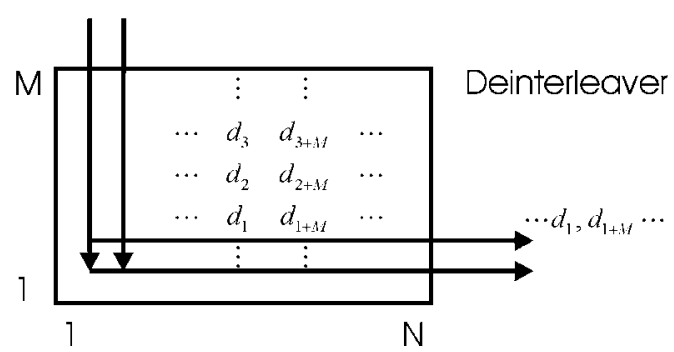

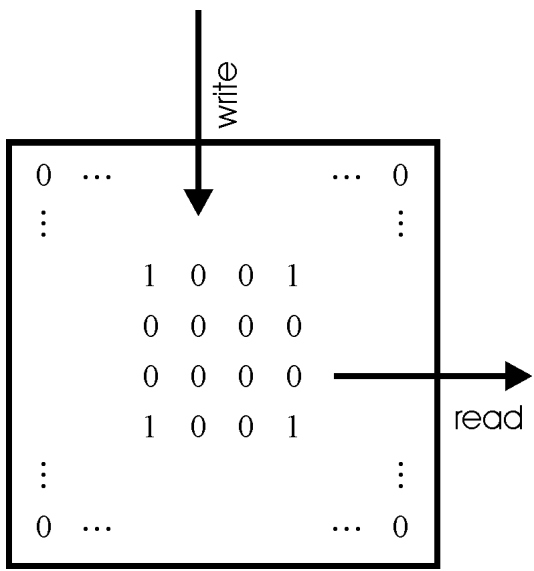

Figure 4: Basic concept of block interleaving (left). A block interleaver fails to break low weight error patterns (right, $1 \equiv$ error, $0 \equiv$ error free symbol).

In the atmospheric optical fading channel, burst error sequences with a mean length of $<\mathrm{DOF}>$ occur. During fades, any information contained in the bit stream can get lost. Due to the high data rates typically used in optical systems and due to the long duration of fades, it is technically not possible to implement block codes longer than the fade duration. However, a code with words shorter than the fade duration can not cope with that situation and the error probability will be higher than without coding. This is an effect of power limited systems, where the added redundancy leads to a lower energy per bit. 
The question arises, how to select the interleaver parameters $\mathrm{N}$ and $\mathrm{M}$, if the interleaver is to be used in the optical fading channel. If the number of columns of the deinterleaver is the same as the block length of the applied code $(\mathrm{N}=\mathrm{n})$, then the deinterleaver should spread error bursts with a length corresponding to $<\mathrm{DOF}>$ to at least $t$ columns, where $t$ is the number of correctable bits in a codeword of length $n$. Hence, the probability of having more than $t$ errors in a word will be small and the depth of such a code matched interleaver should be at least:

$$
M=\left\lceil\frac{\langle D O F\rangle}{t} \cdot \frac{r_{b i t}}{R}\right\rceil
$$

where $r_{b i t}$ is the message data rate. The fade level of the $\left.<\mathrm{DOF}\right\rangle$ is not specified here, since the error performance depends not only on the $<\mathrm{DOF}>$, but also on the link margin and the receiver noise and sensitivity. If the link margin is large, the receiver can deal with deep fades without significant losses.

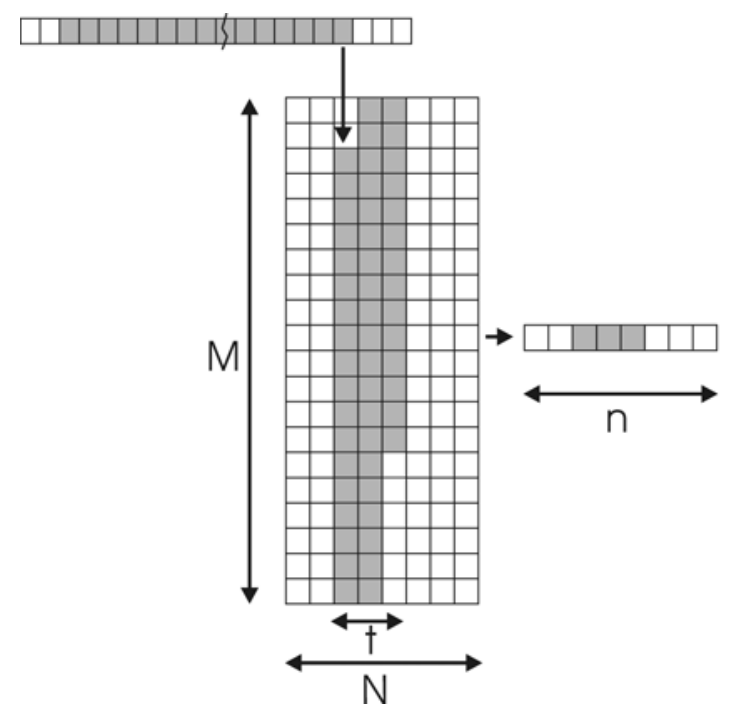

Figure 5: Structure of a deinterleaver with depth $\mathrm{M}$ and a number of columns $\mathrm{N}$ equal to the number of bits $\mathrm{n}$ in the block code codeword. A burst error sequence (grey) is spread over t columns.

\section{SIMULATION MODEL}

To evaluate the channel characteristics, measurements as described above have been done. Data vectors of the received power were taken. The resulting time vectors contain values of received $\mathrm{CW}$ power, which equals to that level a photo detector will receive when sending a one bit in a communication system. The depth of fades in these sampled signals depends on the aperture averaging effect. In our case a $75 \mathrm{~mm}$ collecting aperture is used. More detailed information about the aperture averaging effect can be found in [6].

Due to very slow fading, the channel can be described as frozen and received Rx power is constant for a large amount of bits or even code words. Using an analytical model of the receiver, the instantaneous received power is mapped to an according instantaneous bit error probability. Therefore a certain bit error probability corresponds to each element in the power time vector. Figure 6 displays the performance of the receiver considered here. We used a best fit model of a real receiver, which was used in the frame of the FASOLT experiment [3] for data rates up to 200Mbps. An optimum choice of an adaptive decision threshold, leading to a minimum error probability [8], is assumed. 


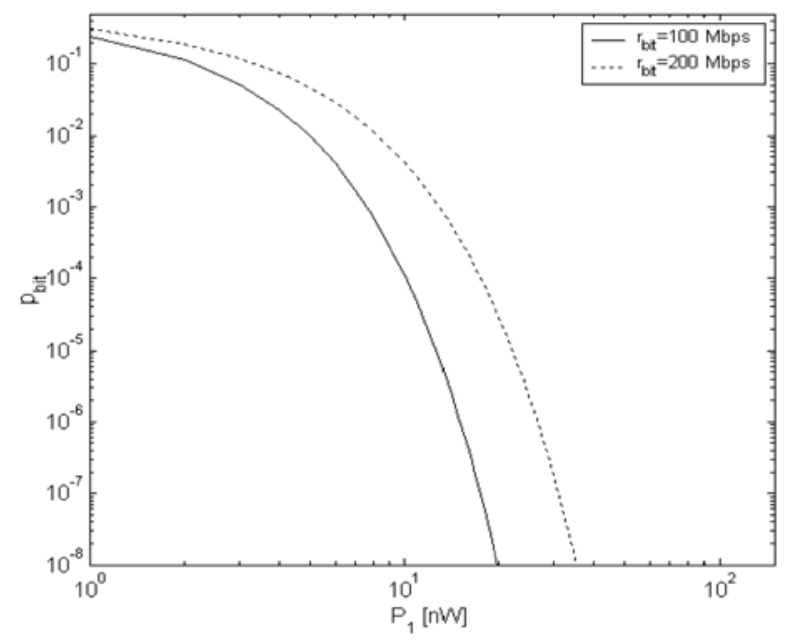

Figure 6: Performance of the considered Rx, bit error probability vs. received peak power (corresponding to a "one" bit)

Our simulations of block coding were all based on this model and the data taken in measurements. Data sampling was done at a rate of $2 \mathrm{ksps}$. Thus, the sampling time $(0.5 \mathrm{msec})$ was much shorter than the minimum fade duration and aliasing can be neglected. The channel behaviour is defined by the power time vectors, which are rescaled to different values of mean power for the simulations. Hence a mean error probability can be calculated for a given mean received power and fading behaviour.

Figure 7 displays the principle of the quasi-analytical model used to calculate an error probability from the power time vectors. First the power vectors are scaled to the appropriate mean value. Then the received power is mapped to a channel error probability using the analytical Rx model. Finally, the uncoded error probability is mapped to an error probability after decoding, using equation (7). For each mean received power value a mean error probability is generated. To check the correctness of the simulation model described above, a bit-wise simulation (Monte Carlo Simulation) has been implemented as well. Both methods lead to consistent results, thus the less computationally expensive analytical method can be used to evaluate block coding problems.

Interleavers are implemented calculating mean values over sections of the error probability vector. An ideal infinite long interleaver will show the mean error probability of the whole error probability vector at its output, since it scrambles the whole sequence.

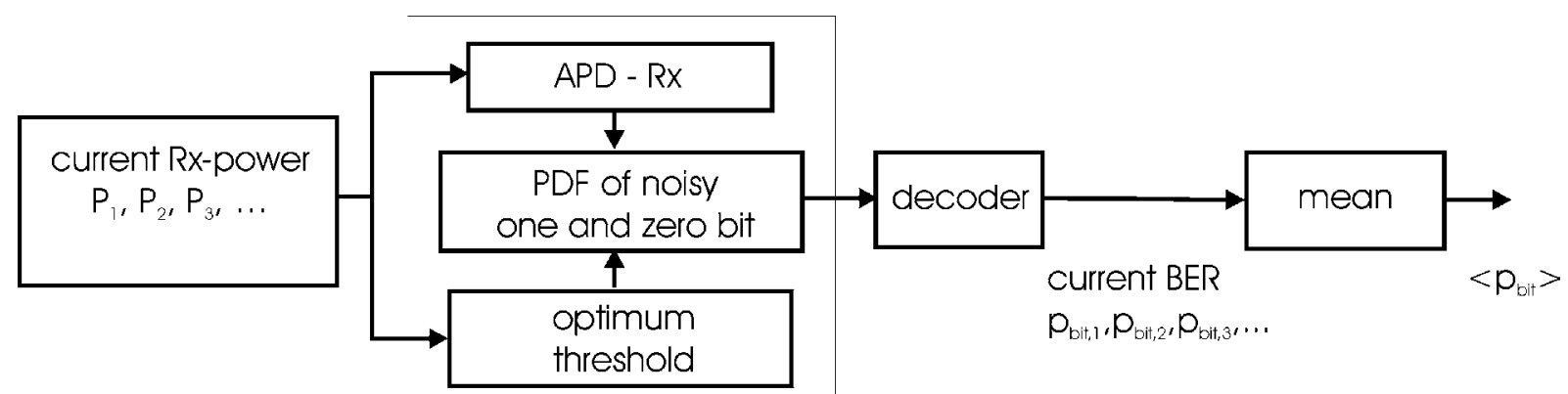

Figure 7: Simulation model of the quasi analytical method 


\section{SIMULATION RESULTS}

\subsection{Simulations using stand alone block codes}

The codes considered here are all binary Bose-Chaudhuri-Hocquenghem or $\mathrm{BCH}(\mathrm{n}, \mathrm{k}, \mathrm{t})$ codes. The simulations are all done for a message data rate of $100 \mathrm{Mbps}$, based on the receiver model described above (for the performance of the Rx see figure 6). The given error probability values are mean values over 65 seconds. Comparing the error probability curves with the curves known for the AWGN channel it can be observed that the intersection of the bit error curves for coded and uncoded transmission appears at very low input error probabilities. To achieve a better performance when using FEC rather than omitting it, there should not be more than t errors in a block of length n. As figure 8 shows for a $(255,131,18)$ code, which can correct up to 18 errors per block, the maximum number of correctable errors if often exceede. Hence this particular code will very often fail for a received power lower than $30 \mathrm{nW}$ (based on the used $\mathrm{Rx}$ model). Generally speaking, block codes are only effective in an optical fading channel for relatively high values of mean received power.

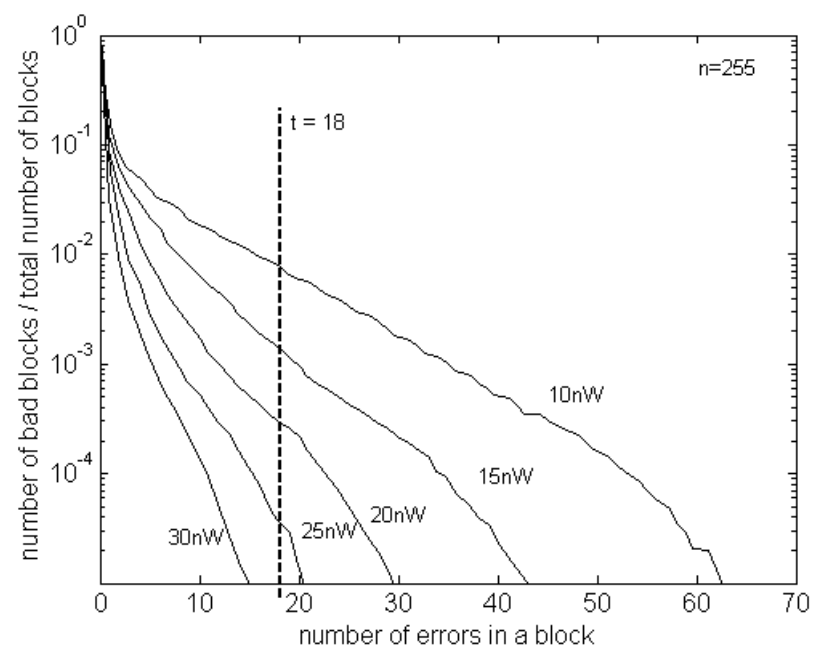

Figure 8: Expected number of errors per block of length 255 for different values of mean received power (channel data rate: $200 \mathrm{Mbps}$, WB scenario)

Figure 9 shows bit error probability vs. mean received power for codes with constant block length in the two considered scenarios Wallberg (WB) and Oberpfaffenhofen (OP). These curves very much depend on the receiver model. Using a more efficient receiver, the curves are moved to a lower received power, however the gradient of the lines will be basically the same. The curves for WB and OP are very similar, but OP is a little worse, due to the higher probability of deep fades (see figure 3). The intercept point of the coded and uncoded transmission is moved to a higher receiver power in the Oberpfaffenhofen scenario. Both plots point out, that codes with a higher redundancy dive faster after the intercept point (with the line for uncoded transmission), however the performance loss for low received power is higher. The performance a the power limited, coded system is only superior to that of an uncoded system, if the code can overcome the effect of lower energy per bit, due to the addition of redundancy.

In figure 10 the error probability for constant rate $\mathrm{BCH}$ block codes is shown. Due to the constant code rate the intercept point is nearly at the same power value for all codes. The behaviour only differs for higher receive powers. If the code rate is constant, the number of correctable bits per block increases with codeword length. That results in a more strong dive of the bit error curve, if the errors occur in bursts. Again the plots reveal that the Oberpfaffenhofen (OP) channel behaves worse, due to the higher probability of deep fades. 

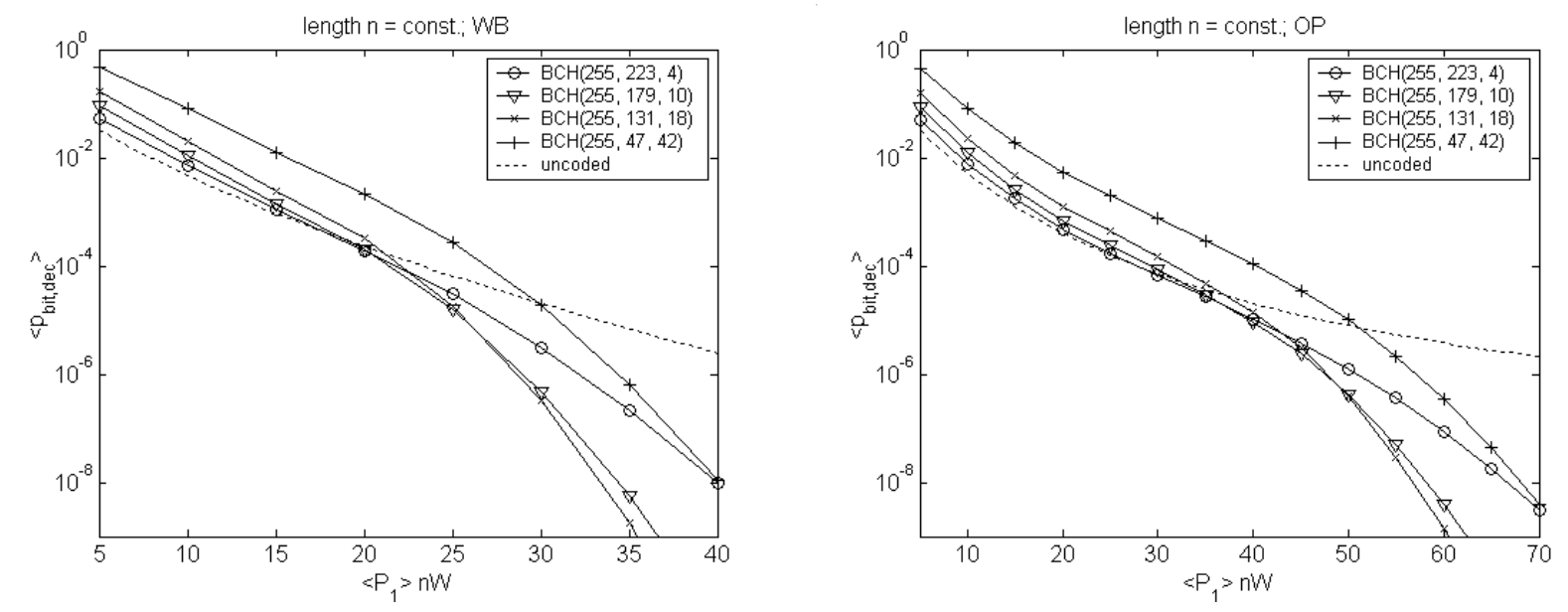

Figure 9: Mean error probability vs. mean received peak power ("one" bit) in the WB (left) and OP (right) scenario for constant block length BCH block codes.
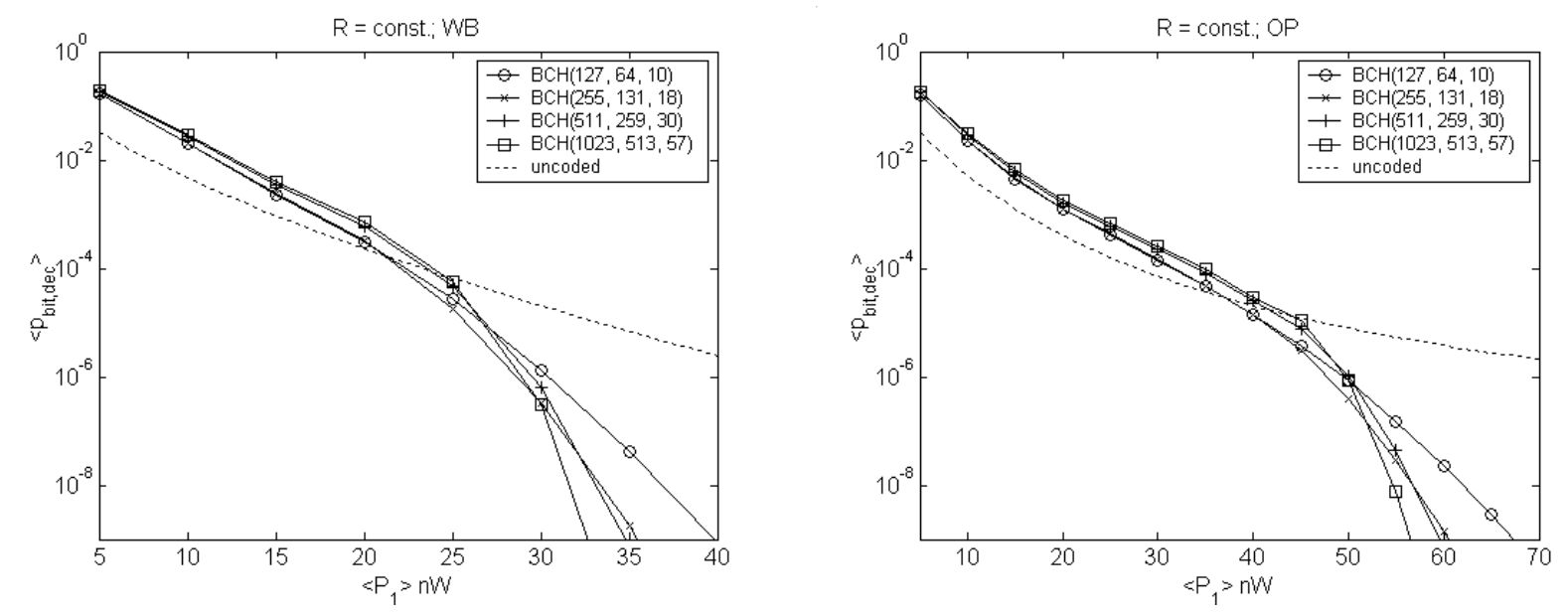

Figure 10: Mean error probability vs. mean received peak power ("one" bit) in the WB (left) and OP (right) scenario for constant rate $\mathrm{BCH}$ block codes

The coding gain (at $\mathrm{p}_{\mathrm{bit}, \mathrm{dec}}=10^{-6}$ ) for block codes is about $1.8 \mathrm{~dB}$ in the WB scenario and $2.0 \mathrm{~dB}$ in the OP scenario, respectively. Much better results can be achieved, when using interleavers, as shown in the next section. The simulations indicate that block codes with low redundancy very often fail, due to deep and extremely long fades (where whole codewords are affected by fades). To improve the system performance, codes which can cope with deep fades are needed. These codes have high redundancy, which results in an unnecessary high channel rate, while errors during the frequent deep fades still can not be corrected. To split the error sequences and always use the whole capability of the code, we also considered code matched interleavers for our simulations.

\subsection{Simulations for combining block codes with interleaving}

Figure 12 displays the effect of interleaving with different interleaver depths, ranging from a 50 kbit depth to an infinite long memory. To show only the influence of interleaving, the same block code ( $\mathrm{BCH}(255,131,18)$, rate one half) is used. To compare the performance of stand alone block codes to coding with interleaving, an additional line is plotted showing only the $\mathrm{BCH}$ code without interleaving. With interleaving the intercept point between coded and uncoded 
transmission is moved to a quite low received power, depending on the interleaver depth. Computing the interleaver depth using (9), the interleaver depth for the WB scenario is $177 \mathrm{kbit}$ using $<\mathrm{DOF} 3 \mathrm{~dB}>$. The curve for a $177 \mathrm{kbit}$ interleaver will be somewhere between the curve of 100 and $200 \mathrm{kbit}$ depth. As the plot shows, the coding gain will be quite acceptable. The optimum of interleaving is shown with an infinite long interleaver. The coding and interleaver (depth $\mathrm{M}=200 \mathrm{kbit}$ ) gain for an error rate of $10^{-6}$ is $3.5 \mathrm{~dB}$ for $\mathrm{WB}$ and $6.5 \mathrm{~dB}$ for OP. This gain is about $3 \mathrm{~dB}$ below the gain with using ideal infinite long interleavers. Comparing interleavers with the same depth in the Wallberg (WB) and Oberpfaffenhofen (OP) scenario, e.g. the 200kbit interleaver is much more effective in the Oberpfaffenhofen scenario due to the shorter duration of fade $\left.\left(\angle \mathrm{DOF} 3 \mathrm{~dB}>_{\mathrm{WB}}=15.9 \mathrm{msec},<\mathrm{DOF} 3 \mathrm{~dB}\right\rangle_{\mathrm{OP}}=2.6 \mathrm{msec}\right)$ in this scenario.
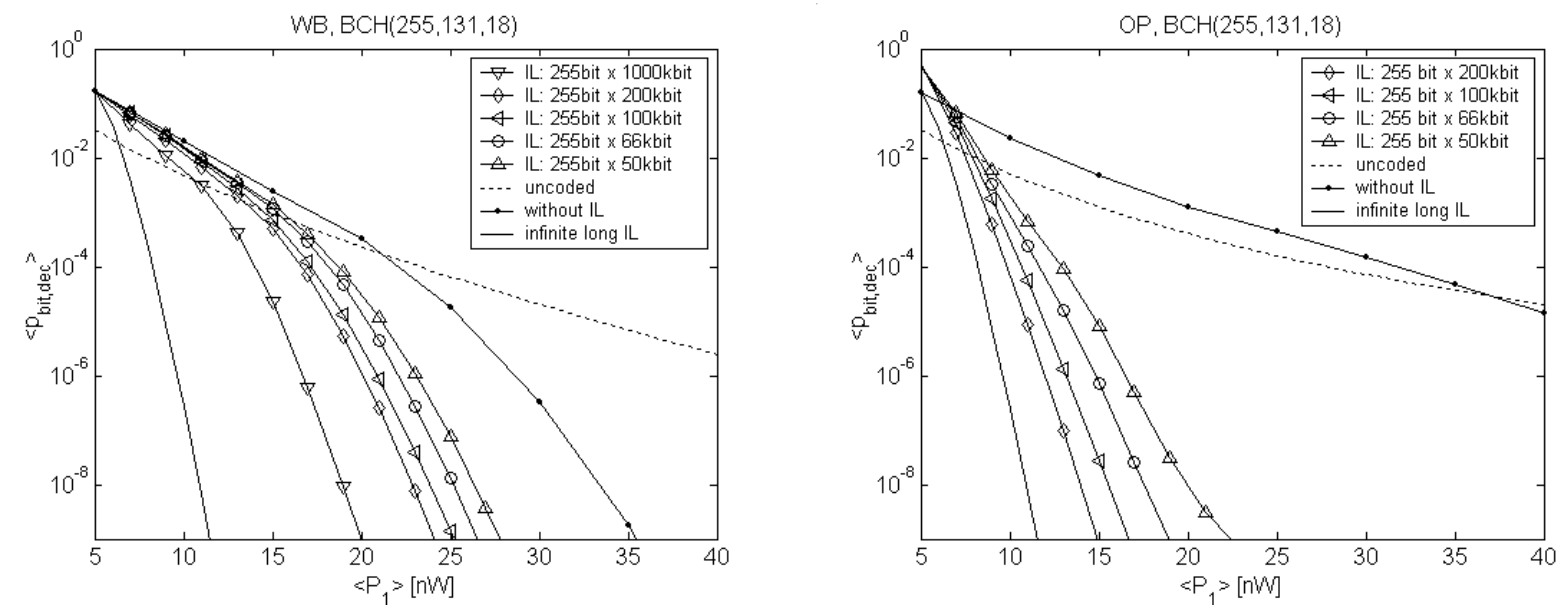

Figure 12: $\mathrm{BCH}(255,131,18)$ block code without and with interleavers of different sizes in the WB scenario (left) and in the OP scenario (right)

Figure 11 shows the combination of a constant depth (200kbit) code matched interleaver with length $\mathrm{N}=\mathrm{n}$ for three different BCH codes. Again it is pointed out, that codes with more redundancy can cope with more errors in the read out sequence of the interleaver and as a result a shorter interleaver can be used for achieving the same mean error ratio. Using the same interleaver and a code with more redundancy will always lead to a better the system performance.

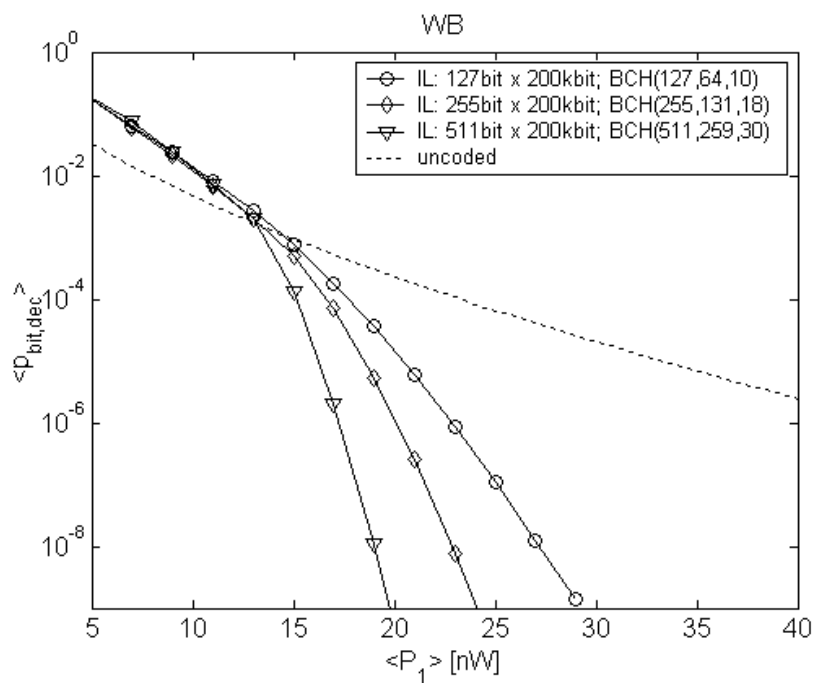

Figure 12: Interleaver with depth $M=200 \mathrm{kbit}$ and a length of $\mathrm{n}=127,255,511$ bit in WB scenario 


\section{SUMMARY AND CONCLUSIONS}

In the atmospheric optical fading channel, the data signal is affected by very slow fading. The mean duration of fades is even much longer than the duration of one block code word. Thus, during fades the code has to deal with many errors distributed over the whole codeword. Therefore the code needs high redundancy and the channel data rate rises unnecessary.

Spreading errors by using a block code matched interleaver will make the block coding more effective. However, when dealing with long fade durations (correlated to the length of error bursts), very long interleavers had to be used. These interleavers often can not be implemented, due to the high demand on memory and the long inherent delay of the interleavers.

Other coding techniques like convolutional codes using soft decision will have to be considered. However, the problem of long deep fades will still exist. Thus coding will not completely solve the problem of fading in the atmospheric optical channel. Other techniques of reducing the impact of fading, such as diversity techniques [7] or aperture averaging [6] have to be considered additionally.

\section{REFERENCES}

[1] D. Cygan: „Ein verkettetes zweistufig- adaptives Fehlersicherungsverfahren für zeitvariable Übertragungskanäle und seine Leistungsfähigkeit im satellitengestützten Landmobilfunk“, VDI Verlag, Düsseldorf, 1991

[2] F. David, D. Giggenbach, et al: "Preliminary results of a $61 \mathrm{~km}$ ground-to-ground optical IM/DD data transmission experiment”, Proc. of SPIE, Vol. 4635, Free-Space Laser Communications Technologies XIV, 2002

[3] F. David, D. Giggenbach, et al: “Overview of the FASOLT experiment and final results”, Proc. of SPIE, Vol. 4975 , Free-Space Laser Communications Technologies XV, 2003

[4] B. Friedrichs: „Kanalcodierung - Grundlagen und Anwendungen in modernen Kommunikationssystemen“, Springer, Berlin, 1996

[5] D. Giggenbach, F. David, et al: "Measurements at a $61 \mathrm{~km}$ near-ground optical transmission channel", Proc. of SPIE, Vol. 4635, Free-Space Laser Communications Technologies XIV, 2002

[6] N. Perlot, H. Bischl, D. Giggenbach: "Discussion of direct detection Rx-power statistics as derived form intensity distribution and comparison with measurements", Proc. of SPIE, Vol. 4976, Atmospheric Propagation, 2003

[7] R. Purvinskis, D. Giggenbach, H. Henniger, N. Perlot: "Multiple wavelength free-space laser communications", Proc. of SPIE, Vol 4975, Free-Space Communications Technologies XV, 2003

[8] S. Alexander: “Optical communication receiver design”, SPIE Press, Bellingham, 1996

[9] B. Vucetic, J. Yuan: “Turbo Codes - Principles and Applications”, Kluwer Academic Publishers, Boston, 2001 